Information of the corresponding author:

First name: Huaxi

Last name: $\mathrm{Gu}$

Email address: $\underline{\text { hxgu@xidian.edu.cn }}$

Phone number: +86-133-5925-2600

Fax number: 86-29-88202524 


\title{
DRTL: a Heat-balanced Deadlock-free Routing Algorithm for 3D Topology
}

\author{
Network-on-Chip \\ Junhui Wang ${ }^{a}$, Huaxi Gu' ${ }^{a}$ Kang Wang ${ }^{a}$, Yintang Yang ${ }^{b}$, Kun Wang , \\ a State Key Laboratory of Integrated Service Networks, Xidian University, Xi'an, China \\ ${ }^{b}$ Institute of Microelectronics, Xidian University \\ c School of Computer Science, Xidian University
}

\begin{abstract}
Heat balance is of critical importance on the design of network-on-chip (NoC). In a 3D topology NoC, routing algorithm should take considerations of each layer's peak temperature and traffic to prolong chip's service life. In this paper, we propose a heat-balanced, deadlock-free routing algorithm named Direct Ratio Transport Layer (DRTL). DRTL distributes and arranges traffics according to the source layer and destination layer in order to achieve heat balance in the chip. In this algorithm, if a transition layer is needed, the probability of choosing a layer as the transition layer is inversely proportional to the distance between this layer and the heat sink. Simulation results showed that, compared with Traffic Aware Downward Routing (TADR), congestion is alleviated and the chip achieves good network performance when DRTL is used. Moreover, DRTL gets almost the same network performance as Transport Layer Assisted Routing (TLAR) does while the chip temperature can be lowered by $2 \mathrm{~K}$.
\end{abstract} Keywords---Heat balance, deadlock-free, routing algorithm, 3D topology

\section{INTRODUCTION}

The International Technology Roadmap of Semiconductors (ITRS) shows that on-chip communication limitations, including energy consumption and signal integrity, constrain the chip performance. 3D integration technology [1], [2], [3] gives chip designers a new dimension of building a chip structure. 3D Network-on-chip (3D NoC) [4], [5] has been proposed as a promising way to solve on-chip communication bottleneck. Topology [6], [7] and routing algorithm [8], [9], [10] have become two important parts of 3D NoC design. In 3D topology architectures [11], [12], [13], [14] different layers are connected using through silicon vias (TSVs) [15], [16]. J. Kim et al. [17] propose a novel partially connected 3D crossbar called DimDe, which is used in 3D stacked mesh architectures. This structure significantly reduces the average latency. DimDe supports a true 3D 
crossbar structure,

Fig.1 Heat conductivity of different layers in 3D NoC

which spans all the active layers of the chip. It also employs a hierarchical arbitration scheme for inter-strata transfers, which reduces area and delay complexity. Marcon César et al. [18] present a Tiny NoC, which is a scalable and efficient 3D mesh architecture developed to minimize latency and NoC area cost. In order to reduce area and lantency, the number of Processing Elements (PEs) is increased on a basic 3D structure based on Through Silicon Via (TSV). Daneshtalab et al. [19] propose two novel clustering stacked topologies for 3D NoC (i.e. CMIT - Clustered Mesh Interlayer Topology; CIT- Concentrated Interlayer Topology) to reduce the area overhead of TSVs and power dissipation on each layer with minimal loss on performance. A. Bose et al. [20] present a new 3D NoC design based on the butterfly fat tree (BFT) topology with an efficient table based uniform routing algorithm. In their architecture, four BFTs are connected together having four root nodes each, and root nodes are connected together to form a complete graph. While 3D integration gives new design space and flexibility of on chip architecture, it also brings challenge in chip thermal management. As the distances between different layers and the heat sink are different, heat conductivity abilities vary. A. Jain et al. [21] and C. H. Chao et al. [22] have proved that under the assumption heat sink removes all the heat of the chip and there is no heat loss in package, layer closest to heat sink is the coolest layer while layer farthest from heat sink is the hottest. Fig.1 shows the temperature distribution of a 3D topology architecture. The bottom layer is close to the heat sink so that it can dissipate heat more easily than the top layer. That means once the chip's traffic is uniform, the heat distribution of layers cannot be uniform. The temperature of top layer will be much higher than that of the bottom layer.

A heat-balanced routing algorithm aims to distribute the heat in an architecture uniformly, which can avoid high peak temperatures in some regions and prolong the service life of the chip. C. H. Chao proposed a traffic and thermal aware routing algorithm called TADR [22]. In TADR, a specified layer is chosen as a transition layer. Packets will first be routed downward to this transition layer. Then these packets will be transmitted on this transition layer and finally routed upward to its destination node. This routing algorithm makes the traffic be close to the heat sink. However, when traffic load increases, more traffic will 
be added to the transition layer, which will cause severe congestion. In addition, TADR is not a shortest path routing algorithm.

Compared with TADR, TLAR [23] makes improvements by choosing transition layer according to a probability distribution.

In TLAR routing algorithm, it is set that every layer between the source node and the destination node has the same probability

to be selected as a transition layer. TLAR achieves better network performance than TADR by reducing the congestion of the first layer and it is a shortest path routing algorithm. But choosing transition layer with equiprobability makes the traffic on the second layer bigger than the bottom layer's in some circumstance, which influence the chip's heat balance performance.

In this paper, it is assumed that there is no heat loss through the packet and the heat sink removes all of the heat. In order to drive more traffic close to the heat sink, we propose a heat-balanced routing algorithm called Direct Ratio Transport Layer (DRTL) routing algorithm. In this algorithm, the probability of choosing a layer as the transition layer is inversely proportional to the distance between this layer and the heat sink. Thus, the layer closer to the heat sink is more likely to be chosen as a transition layer. In addition, DRTL is a shortest path routing algorithm and deadlock-free. It conquers TLAR's shortcomings and achieves almost the same network performance with lower chip temperature.

\section{DRTL ROUTING ALGORITHM}

In a 3D architecture, the core and the interconnect network are the two main parts of energy consumption. Heat generated by data process in cores and data transmission in routers and links contributes to the rising of temperature. When the number of cores increases, the energy consumption of network will be the major part of chip energy consumption. The study of network traffic arrangement will play more and more important role in chip design. Transition layer is used in TADR and TLAR, which helps arrange traffic closer to the heat sink. In our proposed algorithm, transition layer will also be used in some circumstances. According to the relative position between the source node and destination node, DRTL defines three conditions for packets transmission:

Condition 1: The source node is located below the destination node. In this circumstance, DRTL is equal to XYZ[15], [24] 
routing algorithm. The lower layer is much closer to the heat sink and distributing more traffic close to the heat sink benefits the heat balance of all layers.

Condition 2: The source and destination nodes are located on the same layer. In this circumstance, DRTL is equal to XY routing algorithm.

Condition 3: The source node is located above the destination node. In this circumstance, a transition layer is needed. The source node address is defined as $\left(S_{x}, S_{y}, S_{z}\right)$ and the destination node address is defined as $\left(D_{x}, D_{y}, D_{z}\right)$. Each layer from the source layer to the destination layer can be chosen as the transition layer. The set of candidate transition layers is defined as $T=\left\{L_{1}, L_{2}, \ldots, L_{n}\right\}$ where $L_{l}$ is the destination layer, $L_{n}$ is the source layer and $n=S_{z}-D_{z}+1$. Candidate layer $L_{i}$ 's vertical coordinate is $Z_{i}$ and the selection probability of $L_{i}$ is $P_{i}$. The probability of a layer being chosen can be calculated according to equation (1), (2) and (3). These equations indicate that layers which are close to the heat sink are more possible to be chosen as the transition layer.

$$
\begin{gathered}
P_{i}=\frac{\Delta Z_{i}}{\sum_{i=1}^{n} \Delta Z_{i}}, i=1,2,3, \ldots, n \\
Z_{1}=D_{z}, Z_{2}=D_{z}+1, \ldots, Z_{n}=S_{z} \\
\Delta Z_{i}=S_{z}-Z_{i}+1, i=1,2,3, \ldots, n
\end{gathered}
$$

For example, if the source node is on layer 4 and the destination node is on layer 1 . Then $T=\left\{L_{1}, L_{2}, L_{3}, L_{4}\right\} . D_{z}=1$ and $S_{z}=4$.

According to (1), (2) and (3), the vertical coordinate of the four candidate layers are $Z_{1}=1, Z_{2}=2, Z_{3}=3$ and $Z_{4}=4$. $\mathrm{P}_{1}=4 /(4+3+2+1), \mathrm{P}_{2}=3 /(4+3+2+1), \mathrm{P}_{3}=2 /(4+3+2+1)$ and $\mathrm{P}_{4}=1 /(4+3+2+1)$.

The pseudo code of our algorithm is presented as below.

$$
\begin{aligned}
& / * \text { Source node }\left(\mathrm{S}_{\mathrm{x}}, \mathrm{S}_{\mathrm{y}}, \mathrm{S}_{\mathrm{z}}\right) \text {, destination node }\left(\mathrm{D}_{\mathrm{x}}, \mathrm{D}_{\mathrm{y}}, \mathrm{D}_{\mathrm{z}}\right) \text {, current node }\left(\mathrm{C}_{\mathrm{x}}, \mathrm{C}_{\mathrm{y}}, \mathrm{C}_{\mathrm{z}}\right) \text {, the initial value of transition } \\
& \text { layer } \mathrm{L}_{\mathrm{z}}=-1 * / \\
& \Delta \mathrm{X}=D_{x}-C_{x}, \Delta Y=D_{y}-C_{y}, \Delta Z=D_{z}-C_{z} \\
& \Delta Z^{\prime}=D_{z}-S_{z} \\
& \text { if }\left(\Delta Z^{\prime}>0\right) \\
& \quad \text { if }(\Delta X !=0) \\
& \quad \text { route through the } \mathrm{X} \text { dimension until } \Delta \mathrm{X}=0 \\
& \text { else if }(\Delta Y !=0)
\end{aligned}
$$




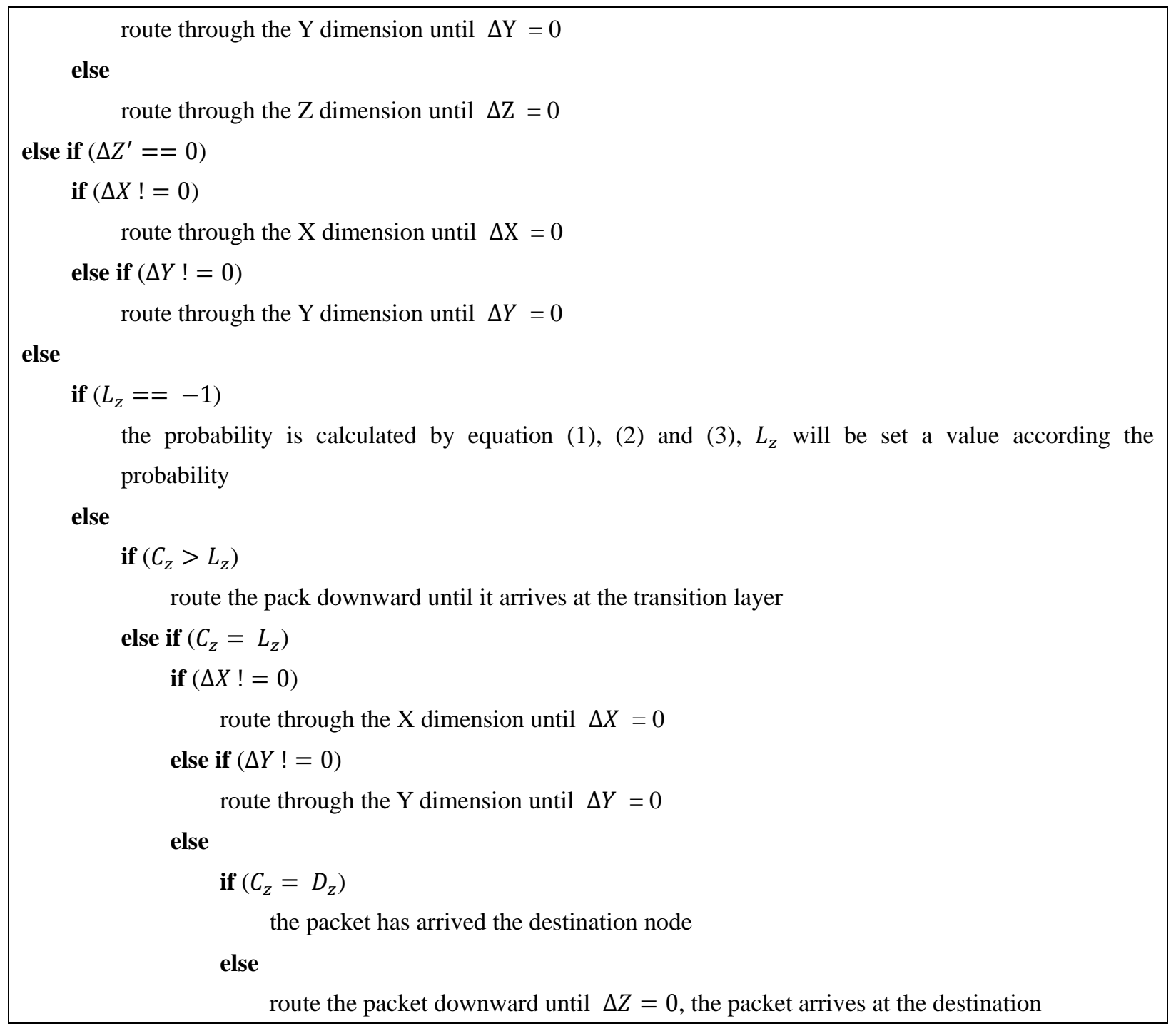

Fig. 2 An example of DRTL routing algorithm in a four-layer 3D NoC.

Fig. 2 shows three DRTL routing examples. Layer 1 is the bottom layer and next to the heat sink. The source nodes are labeled as $S_{1}, S_{2}$ and $S_{3}$. The destination nodes are labeled as $D_{1}, D_{2}$ and $D_{3}$. According to Condition (3), packets sent from $S_{1}$ to $D_{1}$ will be transmitted on a transition layer. In this example, Layer 2 is chosen as the transition layer. According to Condition (2) and (1), packets transmitted from $S_{2}$ to $D_{2}$ are routed on Layer 4 according to $X Y$ routing algorithm and packets sent from $S_{3}$ to $D_{3}$ are routed based on XYZ routing algorithm.

Fig.3 Virtual channel assignment of vertical router in Cluster Mesh topology for a deadlock-free routing. (a) A situation where deadlock occurs when virtual channel is not used. (b) By adding virtual channels $\left(\mathrm{VC}_{1}\right.$ and $\left.\mathrm{VC}_{2}\right)$ in the vertical router input port, deadlock can be avoided. 
Deadlock in a network causes system stall. Channel Dependency Graph (CDG) [25] has been proposed to analyze whether a network is deadlock-free or not. If there is no cyclic dependencies of channels in a network, a routing algorithm will be deadlock-free. Using virtual channels or adopting turn model can prevent a cyclic waiting of buffers in routers. C. Kun-Chih [26] also proposed a method to determine whether a 3D routing algorithm is deadlock-free or not. An deadlock-free algorithm should meet the following two conditions: (1) a deadlock-free routing algorithm is used for horizontal plane communication and (2) when cross layer routing occurs, no packet will be routed upward in vertical direction and then turn on the horizontal plane. We analysis our algorithm based on the packet transmission condition and C. Kun-Chih's work. When packets are routed on the horizontal plane under Condition 1 and Condition 2, XY routing algorithm is used which is deadlock-free. Packets routed upward in Condition 1 are sent to the destination node through TSVs directly and no upward-and-turn routing exists. In Condition 3, packets are routed downward to the transition layer and then to the destination layer. There is no upward-and turn routing either. Based on [26], we draw the conclusion that DRTL routing algorithm is deadlock-free in 3D Mesh [27] and Stack Mesh [28] topologies. In Cluster Mesh [29], four horizontal routers are linked with a vertical router. So packets under Condition 1 will be routed upward to a vertical router and then turn to a horizontal router. The upward-and-turn routed packets and the downward-and-turn routed packets in Cluster Mesh may cause a cyclic buffer dependency in the vertical direction. In this topology, by adding virtual channels at the input ports of a vertical router, DRTL is also deadlock-free. Fig.3 shows the assignment of two virtual channels in a vertical router when Cluster Mesh is used. In this example, when packets are transmitted from this layer's vertical router $\mathrm{VR}_{1}$ to the upper layer's horizontal router $\mathrm{HR}_{1}$, these upward-and-turn packets will be buffered in $\mathrm{VC}_{1}$ and other packets will be buffered in $\mathrm{VC}_{2}$. In this way, a cyclic dependency of input buffer usage is broken.

\section{ALGORITHM PERFORMANCE AND ANALYSIS}

\subsection{Horizontal Traffic Analysis}

In this subsection, we analyze the horizontal traffic loads of each layer under the condition that TADR, TLAR and the proposed 
DRTL routing algorithm are used respectively. Packets transmitted on one layer can be divided into the following four categories: (a)packets produced by this layer and transmitted upward to other layers, (b) packets produced by this layer and transmitted on this certain layer, (c) packets produced by this layer and transmitted downward to other layers and (d) packets produced by other layers and transmitted on this layer. In order to simplify the complexity of calculation, it is assumed that each layer's traffic is uniform and for each packet's transmission, every layer can be the destination layer with the equal probability. Each layer's traffic is calculated considering the traffic coming from all other possible layers. The number of layers is $N$ and traffic produced by each layer is $k$. The horizontal traffic of the $i$ th layer is defined as $S_{i}$.

The calculation methods of $S_{i}$ are given in (4), (9) and (14) when different routing algorithms are used. In TADR's expression, $d$ represents the number of downward routing layers.

TADR:

$$
S_{i}=\left\{\begin{array}{cc}
0, & i>N-d \\
k, & 1<i \leq N-d \\
(d+1) \times k, & i=1
\end{array}\right.
$$

TLAR:

Traffic generated in situation (a):

Traffic generated in situation (b):

Traffic generated in situation (c):

Traffic generated in situation (d):

The total traffic in layer $S_{\mathrm{i}}$ :

$$
S_{i}=\frac{k}{N} \times\left[(n-i)+\sum_{j=1}^{i} \frac{1}{j}+\sum_{t=i+1}^{n} \sum_{s=t-i+1}^{t}\left(\frac{1}{s}\right)\right]
$$

DRTL:

Traffic generated in situation (a):

$$
\frac{k}{N} \times(N-i)
$$

Traffic generated in situation (b):

$$
\frac{k}{N} \times 1
$$

Traffic generated in situation (c):

$$
\frac{k}{N} \times\left(\frac{1}{1+2}+\frac{1}{1+2+3}+\cdots+\frac{1}{1+2+\cdots+i}\right), \mathrm{i} \geq 2
$$


Traffic generated in situation $(\mathrm{d})$ :

$$
\frac{k}{N} \times \sum_{t=i+1}^{n}\left[(t-i+1)\left(\frac{1}{1+2+\cdots+(t-i+1)}+\cdots+\frac{1}{1+2+\cdots+t}\right)\right]
$$

The total traffic in layer $S_{\mathrm{i}}$ :

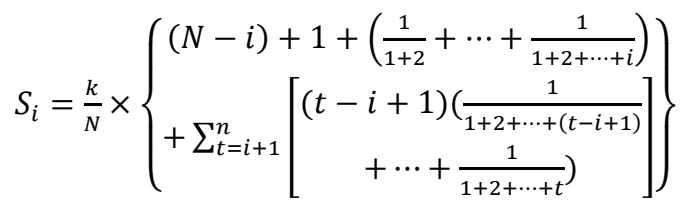

Table. 1 gives the horizontal traffic comparison of the four routing algorithms. TADR's data is achieved when $d=2$. The results show that regardless of the number of layers, TADR only transfers the traffic from top layers to the bottom layer, which causes severe congestion on the bottom layer when the traffic load is high. TLAR algorithm arranges according to the dynamic selection of transition layer. However, when $N=5$, TLAR algorithm makes the second layer's traffic bigger than that of the first layer. Compared with TADR and TLAR, DRTL distributes traffic more reasonable. The traffic increases from the top layer to the bottom layer no matter what the number of layers are, which indicates that DRTL can be widely used in a 3D topology.

\section{TABLE 1 HORIZONTAL TRAFFIC COMPARISON OF DIFFERENT ALGORITHMS}

\subsection{Network Performance and Chip Temperature Analysis}

In this section, we evaluate the performance of the proposed DRTL routing algorithm in both Cluster Mesh and 3D Mesh topologies. Especially the network work performance (such as network latency and network bandwidth) and temperature distribution in different layers. A designed platform based on OPNET and Hotspot [30] are used to get the network end-to-end delay (ETE delay), the throughput and temperature distribution of different layers. The configurations of simulations are listed in

Table 2. We assumed that when a packet is transmitted, each layer has the equal probability to be the destination layer. The temperature simulations take the traffic pattern of each layer, the thermal resistance between nodes and cores power into consideration. The target architecture has 4 layers and 16 cores in each layer. In the simulation, we use the Alpha EV6 core model, the core's function block, area and power consumption parameters are set as the default value that Hotspot provides. By calculating a set of average power values for each of the functional blocks, we evaluate the heat dissipation for all layers of chip 
based on the different routing algorithms. The key information about the proposed chip architecture is shown in Table 3. For comparison, other routing algorithms such as XYZ, TADR and TLAR are also simulated. TADR-N represents a case that packets will be routed downward $\mathrm{N}$ layers in TADR algorithm. For example, TADR-1 means packets will be routed downward one layer. We estimate that each layer has the same heat conductivity and heat sink is the majority structure for heat dissipation when evaluating all algorithms. As to 3D Cluster Mesh topology, virtual channels are needed to avoid deadlock, we use two virtual channels in each router's port.

Fig.4 Simulation results of different algorithms under different traffic models in Cluster Mesh. (a), (b) uniform traffic model. (c), (d) hotspot traffic model. (e), (f) local traffic model.

Fig.4 shows simulation results of different algorithms under different traffic patterns in Cluster Mesh topology. As to network performance such as ETE delay and throughput, XYZ routing algorithm can get better performance than any other algorithms by realizing traffic balance. As TADR transports more traffic to the layer next to the heat sink, it does not perform well in network performance because of severe congestion.

\section{TABLE 2 PARAMETERS AND VALUES OF SIMULATION}

\section{TABLE 3 PARAMETERS AND VALUES IN HOTSPOT}

Comparing DRTL with TLAR, we find they almost have the same throughput performance, which is $85.5 \%, 82.6 \%$ and $88 \%$ that of XYZ algorithm under uniform, hotspot and local traffic models respectively.

Fig.5 depicts temperature simulation results of Cluster Mesh, including the average, the peak and the lowest temperature of each layer. Because of the contradiction between traffic balance and heat balance, though XYZ algorithm gets good network performance, the layers away from heat sink get higher peak temperature than the other algorithm cases. TADR lowers the chip temperature but the first layer's temperature increases dramatically when $\mathrm{N}$ increases. That is because putting more traffic to the first layer induces more heat on this layer, which may outweigh the cooling capacity of the heat sink. Moreover, heat may conduct to the upward layer and that makes other layers' temperatures increase as well. DRTL can reduce the temperature by $2 \mathrm{~K}$ 
compared with TLAR while their network performances are almost the same.

Fig.5 Layers temperature results of different algorithms in Cluster Mesh. (a) peak temperature. (b) lowest temperature. (c) average temperature

As to 3D Mesh topology, Fig.6 and Fig.7 show the simulation results of network performance and chip temperature distribution respectively. The throughput performance of DRTL is $83.3 \%, 91.7 \%$ and $97 \%$ that of XYZ algorithm in three different traffic models and DRTL can reduce chip temperature by $2 \mathrm{~K}$ compared with TLAR.

Considering the implementation processes of DRTL and TLAR, we hold the view that these two algorithm have almost the same cost in transmitting one packet. The only difference between DRTL and TLAR lies in the setting of the possibility. For example, if there are four layers which are possible to be selected as the transition layer, TLAR sets the possibility of each layer to $1 / 4,1 / 4,1 / 4$ and $1 / 4$ while DRTL sets that of each layer to $1 / 10,2 / 10,3 / 10$ and $4 / 10$. By evaluating the traffic distribution feature, the network performance gained and the temperature decrease, we think that the complexity and cost of our algorithm is reasonable. Moreover, though the simulations are based on uniform network, our algorithm can perform good heat balance in a more realistic non-fully connected network by working alone or cooperated with other non-uniform network algorithm. In the case of working alone, a non-uniform network is like a Cluster Mesh topology while the number of nodes (horizontal routers) connecting with a vertical router is no longer the same. As to a combination usage case with other algorithms, for example, our algorithm can work together with Elevator-first which has been proved perform well in non-uniform network. The selection of the transition layer can be done based on our proposed algorithm and vertical link selection can be done based on the Elevator-first algorithm.

Fig.6 Simulation results of different algorithms under different traffic models in 3D Mesh. (a), (b) uniform traffic model. (c), (d) hotspot traffic model. (e), (f) local traffic model

Fig.7 Layers temperature results of different algorithms in 3D Mesh. (a) peak temperature. (b) lowest temperature. (c) average temperature

\section{Conclusion}

In this paper, a heat-balanced deadlock-free routing algorithm named DRTL is proposed. In this routing algorithm, a transition layer is selected for distributing more traffic to the layers close to the heat sink. The probability of a layer being chosen as a transition layer is associated with the distance between the source and the destination node. DRTL acquires a good tradeoff 
between network performance and chip temperature. Under almost the same cost as TLAR, the proposed algorithm ensures the whole can work at a lower temperature, which will prolong the chip's service life. Besides, our research also shows that by adjusting the selection probability of transition layer according to the heat dissipation model, the chip can gain better heat balance performance. Compared with TLAR, we hold the view that there will be quite small hardware changes in the realization of DRTL. And we think the increased hardware overhead is worthwhile for a more reasonable temperature distribution between different layers. Simulation results of different topologies confirm that DRTL routing algorithm is of good portability. It can be expected that by applying chip mapping optimization and increasing TSV's heat emission, the result of our proposed DRTL routing algorithm can be better. The detailed hardware overhead analysis of DRTL will be given in our future work.

\section{Acknowledgments}

This work is supported by the National Science Foundation of China Grant No. 61472300 and 61334003, the Fundamental Research Funds for the Central Universities Grant No. JB150318, the 111 Project Grant No.B08038.

\section{Reference}

[1] H. H. S. Lee and K. Chakrabarty, "Test Challenges for 3D Integrated Circuits," Design \& Test of Computers, IEEE, vol. 26, pp. 26-35, 2009.

[2] P. Ramm, A. Klumpp, J. Weber, N. Lietaer, M. Taklo, W. De Raedt, et al., "3D Integration technology: Status and application development," in ESSCIRC, 2010 Proceedings of the, 2010, pp. 9-16.

[3] M. Koyanagi, L. Kang-Wook, T. Fukushima, and T. Tanaka, "Heterogeneous 3D integration technology and new 3D LSIs," in Solid-State and Integrated Circuit Technology (ICSICT), 2012 IEEE 11th International Conference on, 2012, pp. 1-4.

[4] A. W. Yin, T. C. Xu, Y. Bo, P. Liljeberg, and H. Tenhunen, "Change Function of 2D/3D Network-on-Chip," in Computer and Information Technology (CIT), 2011 IEEE 11th International Conference on, 2011, pp. 181-188.

[5] A. M. Rahmani, K. R. Vaddina, K. Latif, P. Liljeberg, J. Plosila, and H. Tenhunen, "Design and management of high-performance, reliable and 
thermal-aware 3D networks-on-chip," Circuits, Devices \& Systems, IET, vol. 6, pp. 308-321, 2012.

[6] V. F. Pavlidis and E. G. Friedman, "3-D Topologies for Networks-on-Chip," in SOC Conference, IEEE International, 2006, pp. $285-288$.

[7] A. M. Rahmani, K. R. Vaddina, K. Latif, P. Liljeberg, J. Plosila, and H. Tenhunen, "Generic Monitoring and Management Infrastructure for 3D NoC-Bus Hybrid Architectures," in Networks on Chip (NoCS), 2012 Sixth IEEE/ACM International Symposium on, 2012, pp. 177-184.

[8] F. Dubois, A. Sheibanyrad, Pe, x, F. trot, and M. Bahmani, "Elevator-First: A Deadlock-Free Distributed Routing Algorithm for Vertically Partially Connected 3D-NoCs," Computers, IEEE Transactions on, vol. 62, pp. 609-615, 2013.

[9] S. Akbari, A. Shafiee, M. Fathy, and R. Berangi, "AFRA: A low cost high performance reliable routing for 3D mesh NoCs," in Design, Automation \& Test in Europe Conference \& Exhibition (DATE), 2012, pp. 332-337.

[10] X. Dong, "Deadlock-Free Adaptive Routing in Meshes with Fault-Tolerance Ability Based on Channel Overlapping," Dependable and Secure Computing, IEEE Transactions on, vol. 8, pp. 74-88, 2011.

[11] V. F. Pavlidis and E. G. Friedman, "3-D Topologies for Networks-on-Chip," Very Large Scale Integration (VLSI) Systems, IEEE Transactions on, vol. 15, pp. 1081-1090, 2007.

[12] B. S. Feero and P. P. Pande, "Networks-on-Chip in a Three-Dimensional Environment: A Performance Evaluation," Computers, IEEE Transactions on, vol. 58, pp. 32-45, 2009.

[13] R. W. Morris, A. K. Kodi, A. Louri, and R. D. Whaley, "Three-Dimensional Stacked Nanophotonic Network-on-Chip Architecture with Minimal Reconfiguration," Computers, IEEE Transactions on, vol. 63, pp. 243-255, 2014.

[14] R. S. Ramanujam and L. Bill, "A Layer-Multiplexed 3D On-Chip Network Architecture," Embedded Systems Letters, IEEE, vol. 1, pp. 50-55, 2009.

[15] A. B. Ahmed and A. B. Abdallah, "LA-XYZ: Low Latency, High Throughput Look-Ahead Routing Algorithm for 3D Network-on-Chip (3D-NoC) Architecture," in Embedded Multicore Socs (MCSoC), 2012 IEEE 6th International Symposium on, 2012, pp. 167-174.

[16] T. C. Xu, P. Liljeberg, and H. Tenhunen, "A study of Through Silicon Via impact to 3D Network-on-Chip design," in Electronics and Information Engineering (ICEIE), 2010 International Conference On, 2010, pp. V1-333-V1-337.

[17] Kim, J., Nicopoulos, C., Park, D., Das, R., Xie, Y., Narayanan, V., . \& Das, C. R., “A novel dimensionally-decomposed router for on-chip communication in 3D architectures," In ACM SIGARCH Computer Architecture News, Vol. 35, pp. 138-149, 2007 
[18] Marcon, C., Fernandes, R., Cataldo, R., Grando, F., Webber, T., Benso, A., \& Poehls, L. B, "Tiny NoC: a 3D mesh topology with router channel optimization for area and latency minimization," VLSI Design and Embedded Systems, 27th International Conference on, 2014 , pp. $228-233$.

[19] Daneshtalab, M., Ebrahimi, M., Liljeberg, P., Plosila, J., \& Tenhunen, “Cluster-based topologies for 3D stacked architectures,” In Proceedings of the 8th ACM International Conference on Computing Frontiers, 2011, pp. 14.

[20] Bose, Anjan, Prasun Ghosal, and Saraju P. Mohanty. "A low latency scalable 3D NoC using BFT topology with table based uniform routing." VLSI (ISVLSI), IEEE Computer Society Annual Symposium on, 2014, pp. 136-141.

[21] A. Jain, R. E. Jones, R. Chatterjee, S. Pozder, "Analytical and Numerical Modeling of the Thermal Performance of Three-Dimensional Integrated Circuits," IEEE Transactions on Components and Packaging Technologies, vol.33, no.1, 2010, pp.56-63

[22] C. Chih-Hao, J. Kai-Yuan, W. Hao-Yu, W. Jia-Cheng, and W. An-Yeu, "Traffic- and Thermal-Aware Run-Time Thermal Management Scheme for 3D NoC Systems," in Networks-on-Chip (NOCS), 2010 Fourth ACM/IEEE International Symposium on, 2010, pp. 223-230.

[23] C. Chih-Hao, Y. Tsu-Chu, L. Shu-Yen, and W. An-Yeu, "Transport Layer Assisted Routing for Non-Stationary Irregular mesh of thermal-aware 3D Network-on-Chip systems," in SOC Conference (SOCC), 2011 IEEE International, 2011, pp. 284-289..

[24] J. Flich, S. Rodrigo, and J. Duato, "An Efficient Implementation of Distributed Routing Algorithms for NoCs," in Networks-on-Chip, 2008. NoCS 2008. Second ACM/IEEE International Symposium on, 2008, pp. 87-96.

[25] W. J. Dally and C. L. Seitz, "Deadlock-Free Message Routing in Multiprocessor Interconnection Networks," Computers, IEEE Transactions on, vol. C-36, pp. 547-553, 1987.

[26] C. Kun-Chih, C. Chih-Hao, L. Shu-Yen, H. Hui-Shun, and W. An-Yeu, "Transport-layer assisted vertical traffic balanced routing for thermal-aware three-dimensional Network-on-Chip systems," in VLSI Design, Automation, and Test (VLSI-DAT), 2012 International Symposium on, 2012 , pp. 1-4.

[27] B. S. Feero and P. P. Pande, "Networks-on-Chip in a Three-Dimensional Environment: A Performance Evaluation," Computers, IEEE Transactions on, vol. 58, pp. 32-45, 2009.

[28] L. Feihul, C. Nicopoulos, T. Richardson, X. Yuan, V. Narayanan, and M. Kandemir, "Design and Management of 3D Chip Multiprocessors Using Network-in-Memory," in Computer Architecture, 2006. ISCA '06. 33rd International Symposium on, 2006, pp. 130-141.

[29] J. Wang, H. Gu, Y. Yang, “Cluster mesh: a topology for three-dimensional network-on-chip,” IEICE Electronics Express, vol. 9, no. 15, pp. 1254-1259, 2012. 
[30] H. Wei, S. Ghosh, S. Velusamy, K. Sankaranarayanan, K. Skadron, and M. R. Stan, "HotSpot: a compact thermal modeling methodology for early-stage VLSI design," Very Large Scale Integration (VLSI) Systems, IEEE Transactions on, vol. 14, pp. 501-513, 2006. 


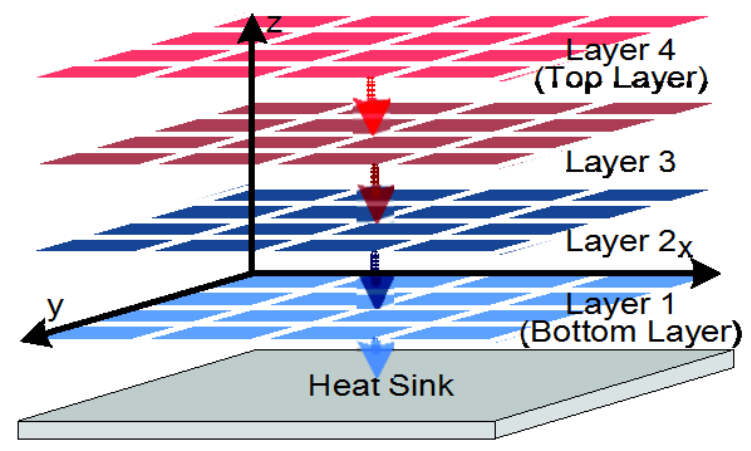

Fig.1 Heat conductivity of different layers in 3D NoC 


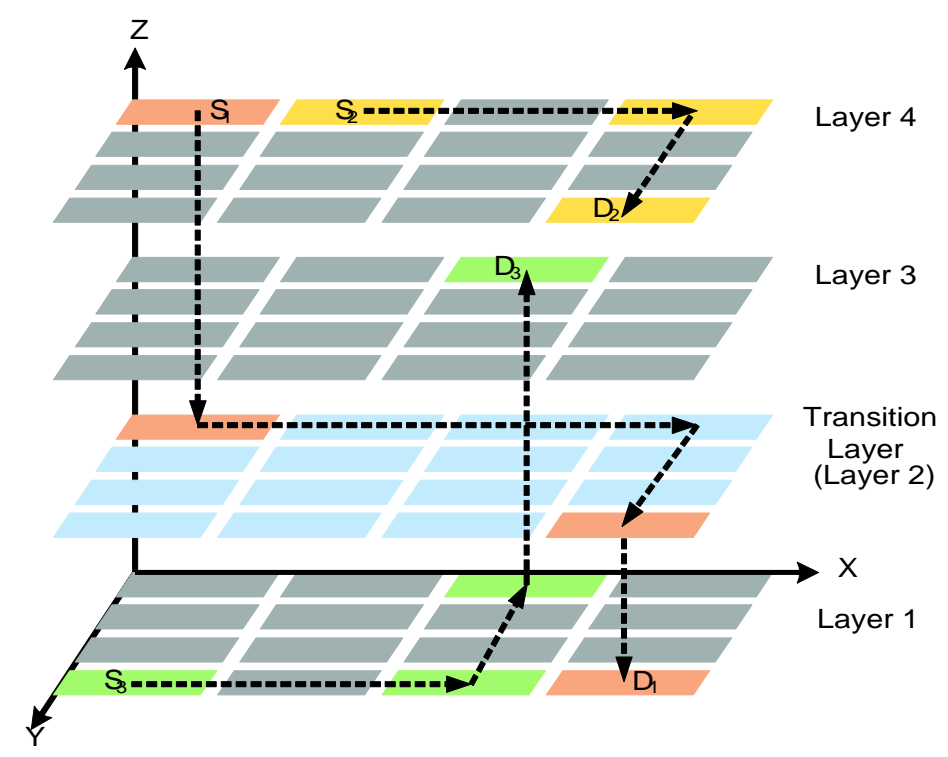

Fig. 2 An example of DRTL routing algorithm in a four-layer 3D NoC. 

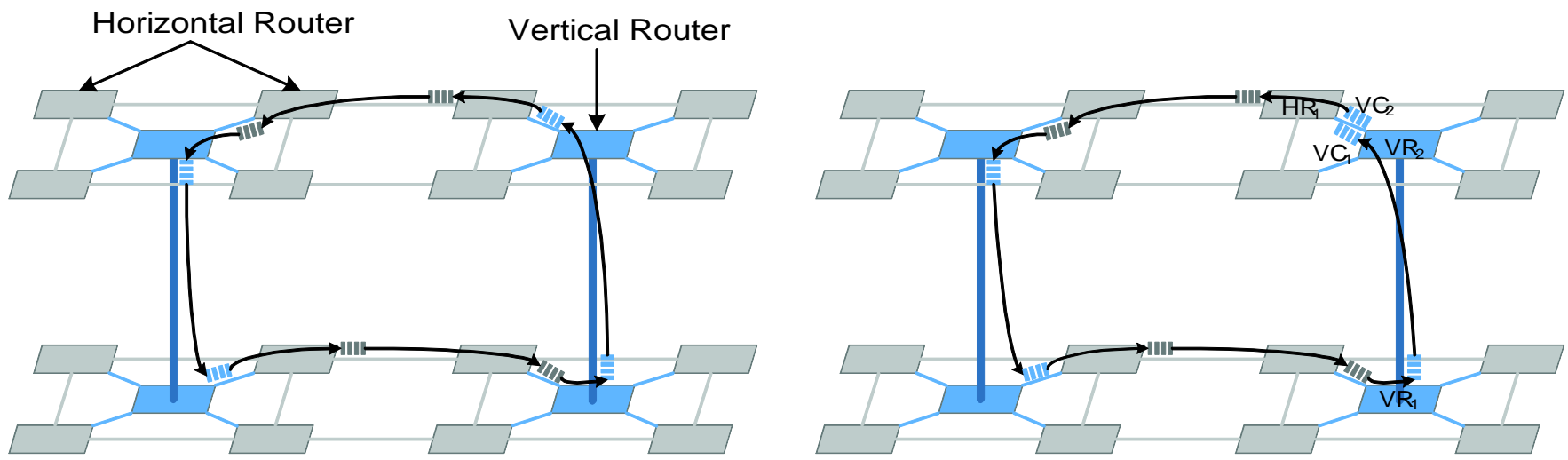

(a)(b)

Fig.3 Virtual channel assignment of vertical router in Cluster Mesh topology for a deadlock-free routing. (a) A situation where deadlock occurs when virtual channel is not used. (b) By adding virtual channels $\left(\mathrm{VC}_{1}\right.$ and $\left.\mathrm{VC}_{2}\right)$ in the vertical router input port, deadlock can be avoided. 


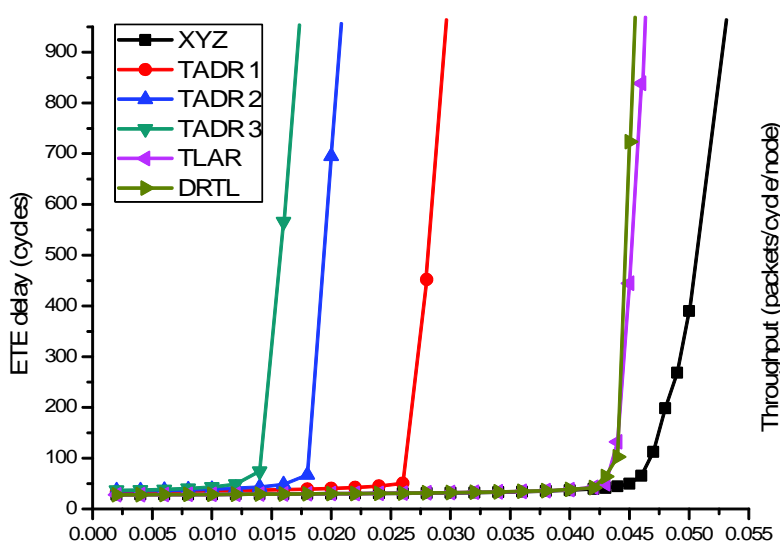

Offered load (packets/cycle/node)

(a)

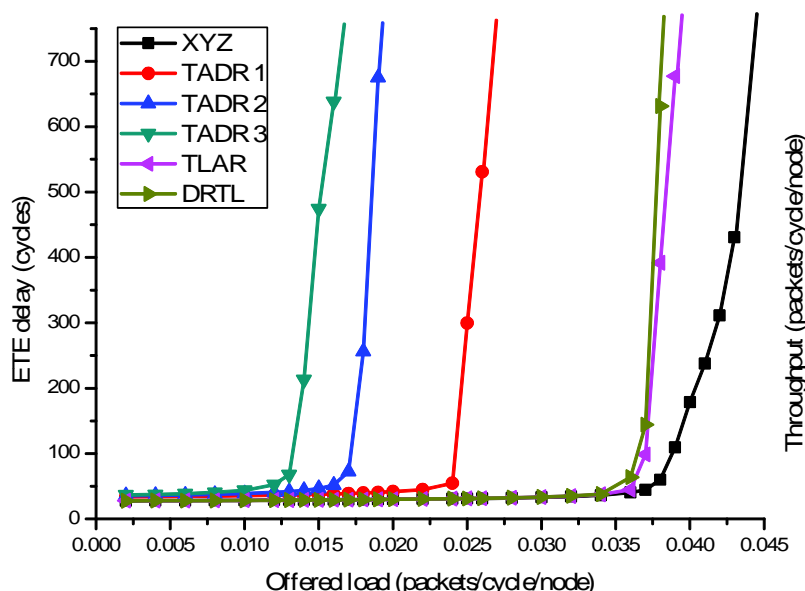

Offered load (packets/cycle/node)

(d)

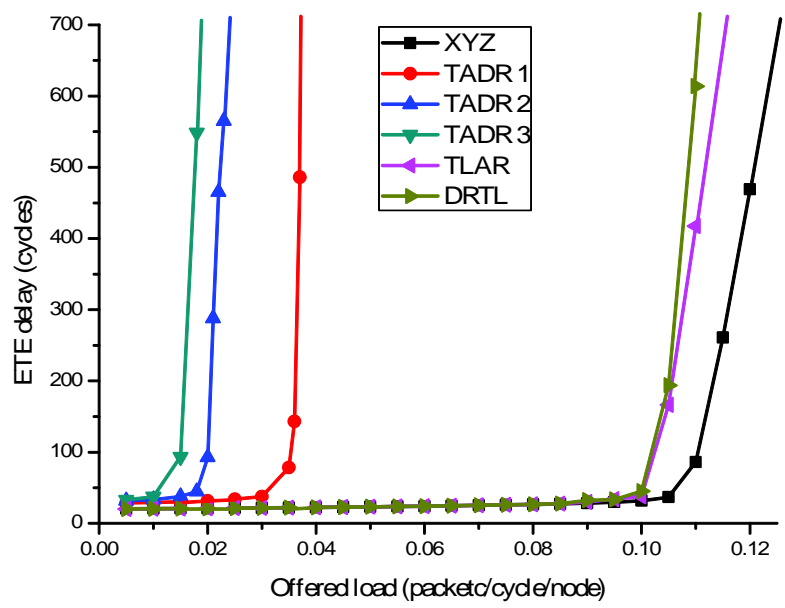

(e)

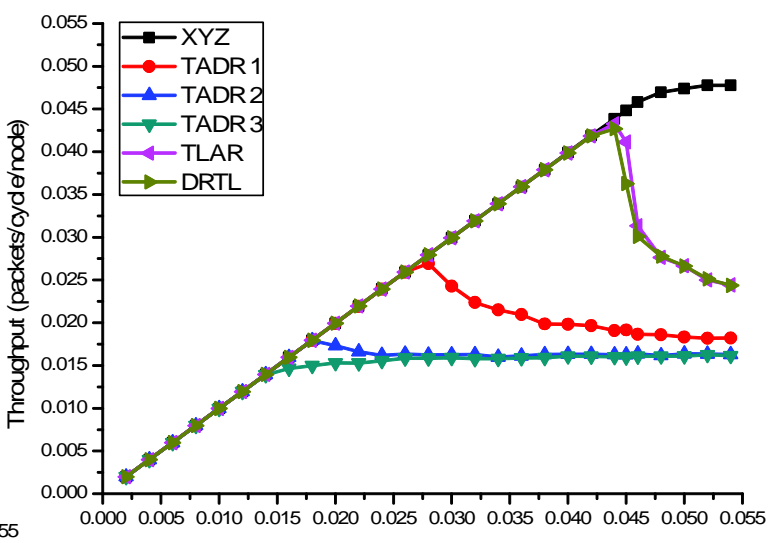

Offered load (packets/cycle/node)

(b)

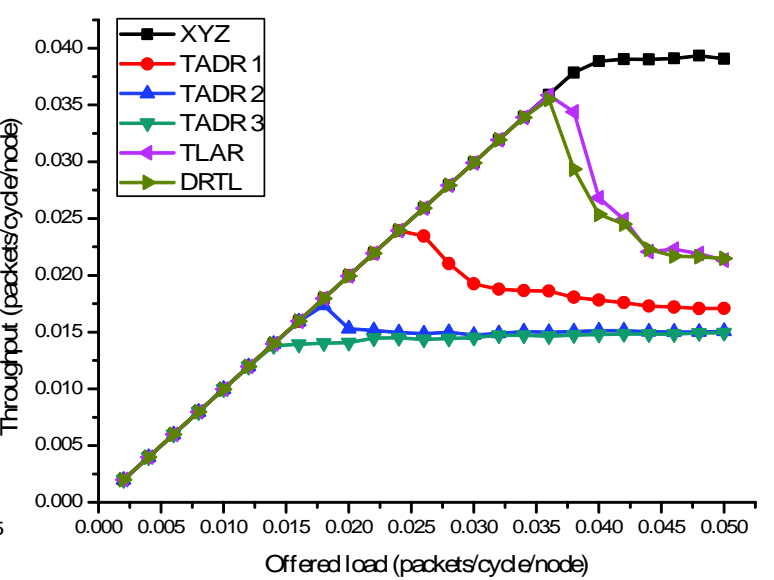

(e)

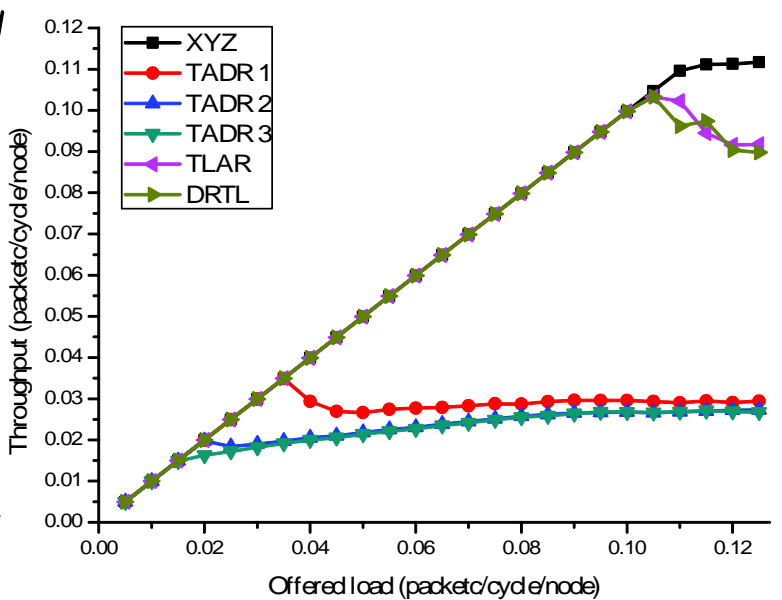

(f)

Fig.4 Simulation results of different algorithms under different traffic models in Cluster Mesh. (a), (b) uniform traffic model. (c), (d) hotspot traffic model. (e), (f) local traffic model. 


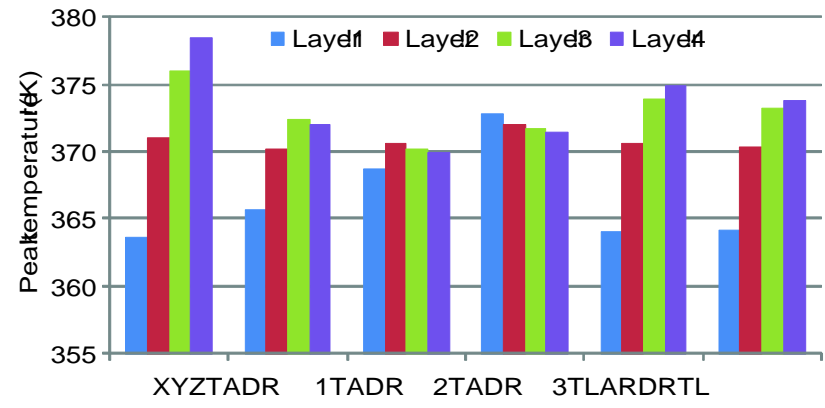

(a)

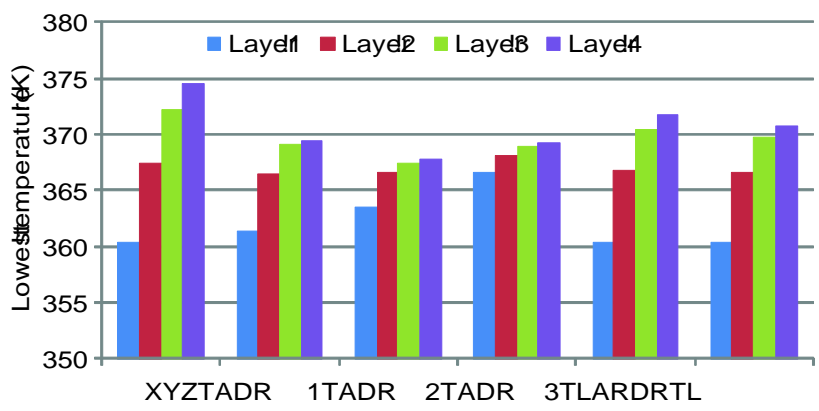

(b)

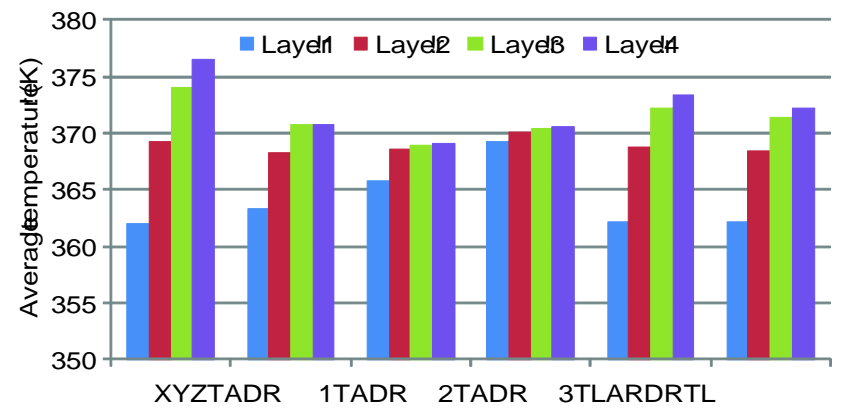

(c)

Fig.5 Layers temperature results of different algorithms in Cluster Mesh. (a) peak temperature. (b) lowest temperature. (c) average temperature 


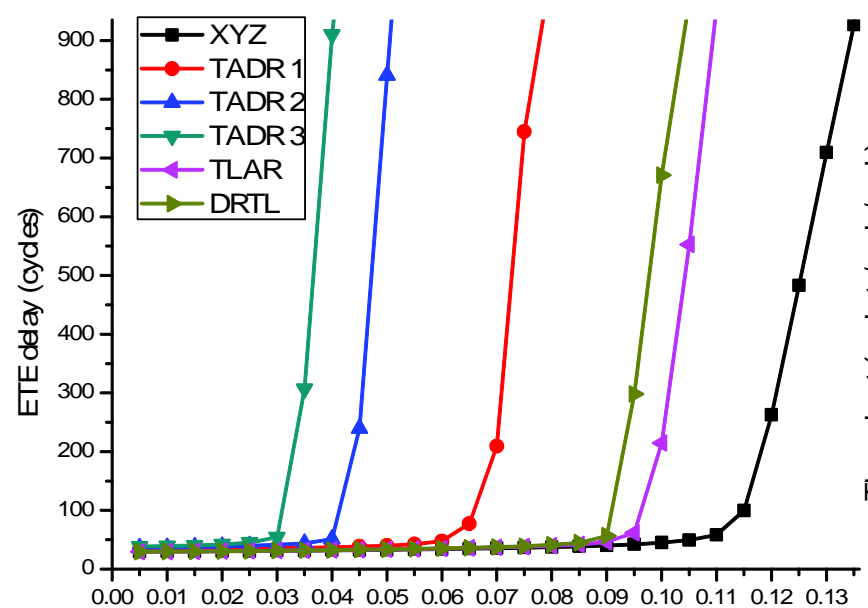

Offered load (packets/cycle/node)

(a)

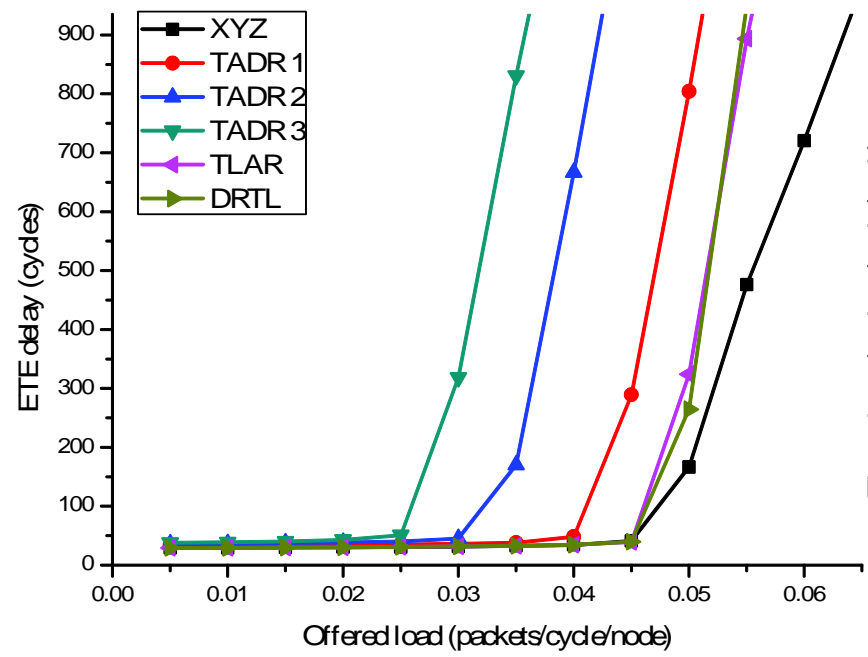

(d)

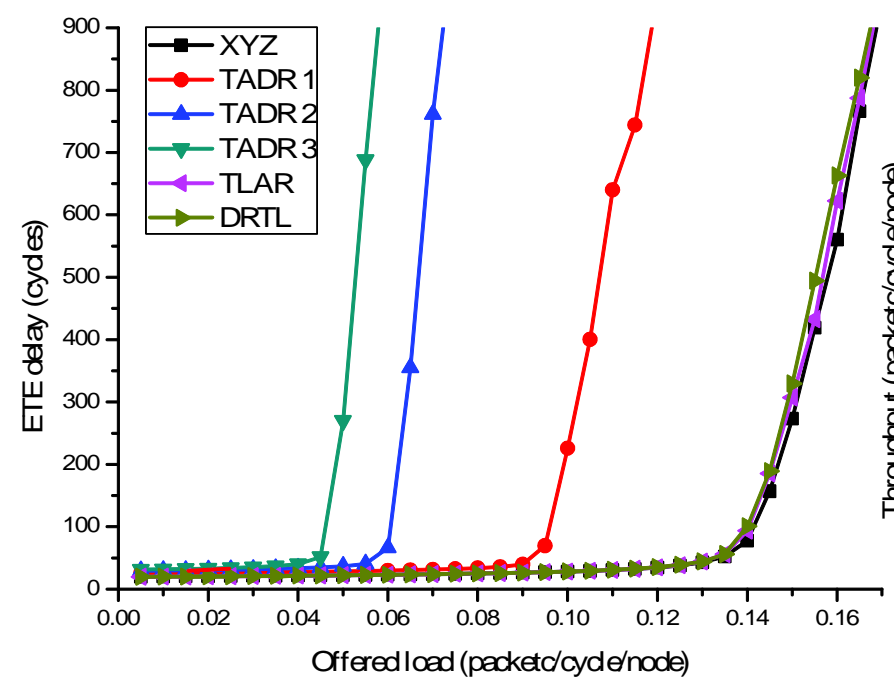

(e)

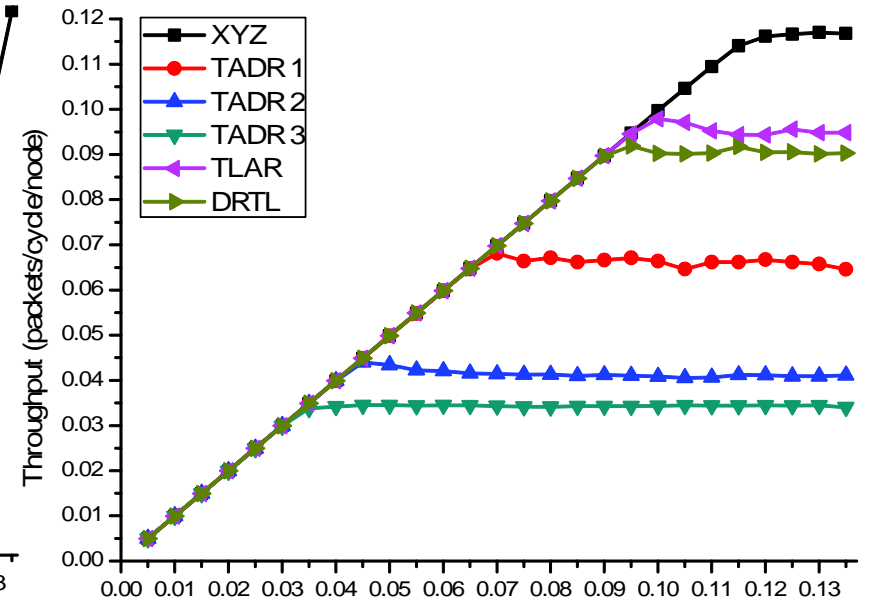

Offered load (packets'cycle/node)

(b)

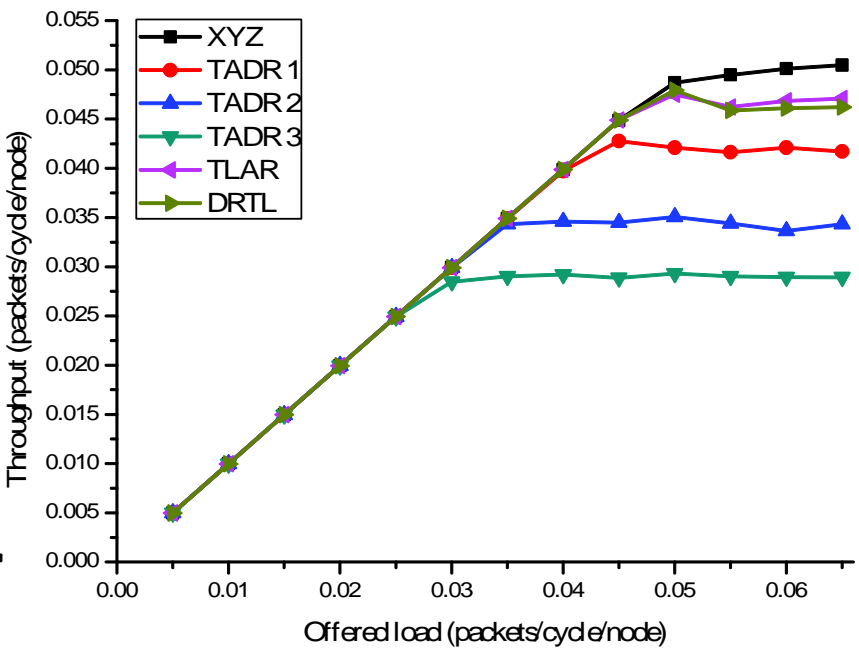

(e)

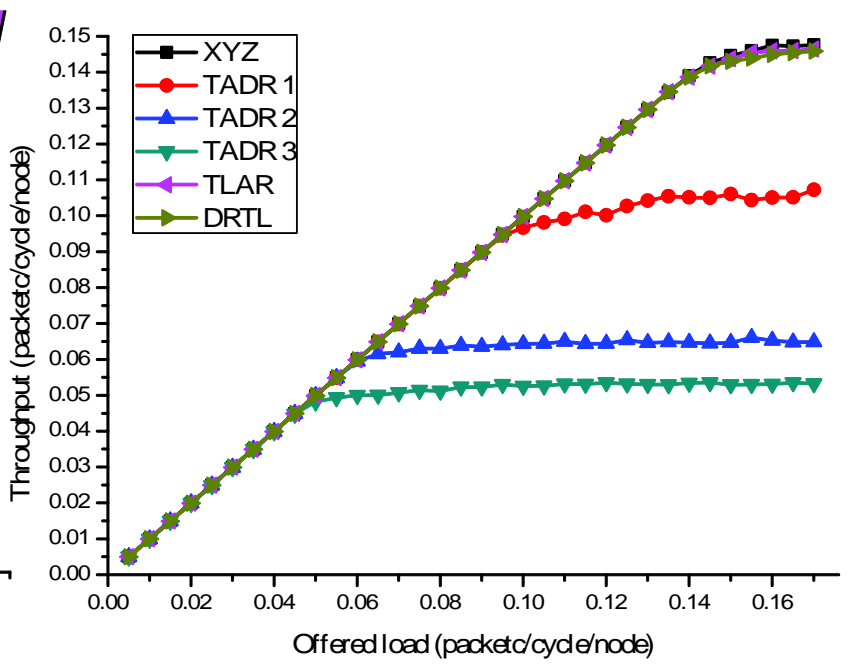

(f)

Fig.6 Simulation results of different algorithms under different traffic models in 3D Mesh. (a), (b) uniform traffic model. (c), (d) hotspot traffic model. (e), (f) local traffic model 


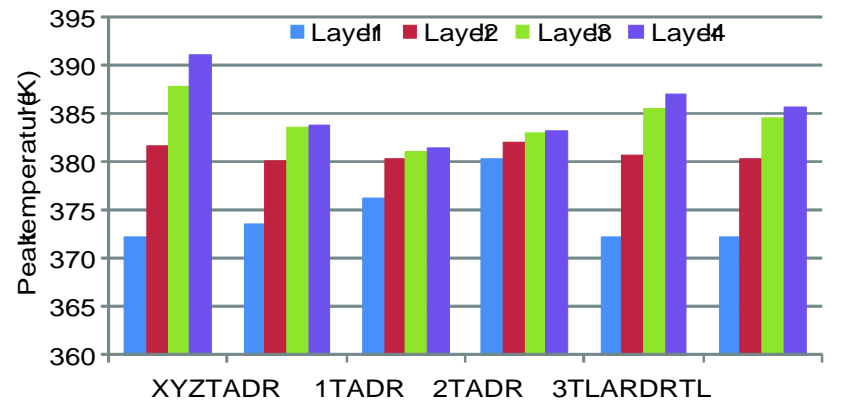

(a)

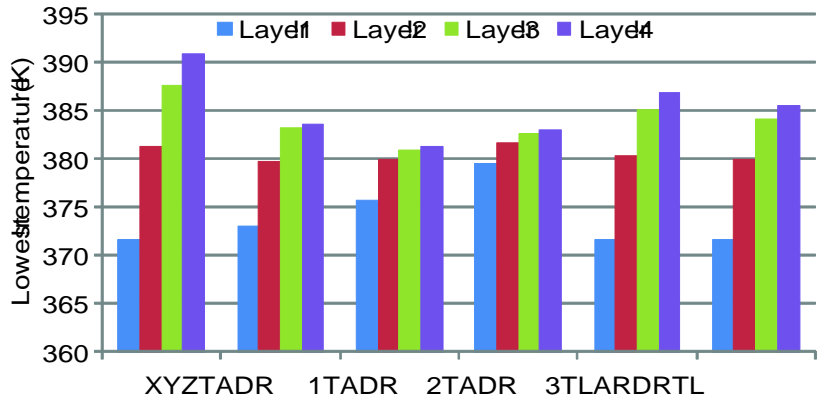

(b)

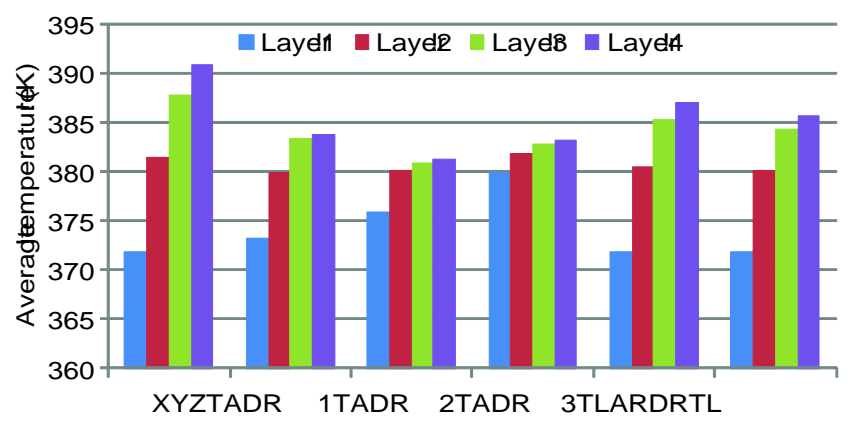

(c)

Fig.7 Layers temperature results of different algorithms in 3D Mesh. (a) peak temperature. (b) lowest temperature. (c) average temperature 
TABLE 1 HORIZONTAL TRAFFIC COMPARISON OF DIFFERENT ALGORITHMS

\begin{tabular}{|c|c|c|c|c|c|c|c|c|}
\hline $\mathrm{N}$ & $\mathrm{i}$ & $\mathrm{XYZ}$ & TADR & TLAR & DRTL & $\begin{array}{c}\text { Traffic amplification } \\
\text { in TADR }\end{array}$ & $\begin{array}{c}\text { Traffic amplification } \\
\text { in TLAR }\end{array}$ & $\begin{array}{c}\text { Traffic amplification } \\
\text { in DRTL }\end{array}$ \\
\hline \multirow{3}{*}{3} & 1 & $\mathrm{k}$ & $3 \mathrm{k}$ & $23 \mathrm{k} / 18$ & $25 \mathrm{k} / 18$ & $200 \%$ & $27.8 \%$ & $38.9 \%$ \\
\hline & 2 & $\mathrm{k}$ & 0 & $20 \mathrm{k} / 18$ & $20 \mathrm{k} / 18$ & $-100 \%$ & $11.1 \%$ & $11.1 \%$ \\
\hline & 3 & $\mathrm{k}$ & 0 & $11 \mathrm{k} / 18$ & $9 \mathrm{k} / 18$ & $-100 \%$ & $-38.9 \%$ & $-50 \%$ \\
\hline \multirow{4}{*}{4} & 1 & $\mathrm{k}$ & $3 \mathrm{k}$ & $61 \mathrm{k} / 48$ & $167 \mathrm{k} / 120$ & $200 \%$ & $27.1 \%$ & $39.2 \%$ \\
\hline & 2 & $\mathrm{k}$ & $\mathrm{k}$ & $59 \mathrm{k} / 48$ & $154 \mathrm{k} / 120$ & 0 & $22.9 \%$ & $28.3 \%$ \\
\hline & 3 & $\mathrm{k}$ & 0 & $47 \mathrm{k} / 48$ & $111 \mathrm{k} / 120$ & $-100 \%$ & $-2.1 \%$ & $-7.5 \%$ \\
\hline & 4 & $\mathrm{k}$ & 0 & $25 \mathrm{k} / 48$ & $48 \mathrm{k} / 120$ & $-100 \%$ & $-47.9 \%$ & $-60 \%$ \\
\hline \multirow{5}{*}{5} & 1 & $\mathrm{k}$ & $3 \mathrm{k}$ & $377 \mathrm{k} / 300$ & $207 \mathrm{k} / 150$ & $200 \%$ & $25.7 \%$ & $38 \%$ \\
\hline & 2 & $\mathrm{k}$ & $\mathrm{k}$ & $382 \mathrm{k} / 300$ & $204 \mathrm{k} / 150$ & $0 \%$ & $27.3 \%$ & $36 \%$ \\
\hline & 3 & $\mathrm{k}$ & $\mathrm{k}$ & $342 \mathrm{k} / 300$ & $171 \mathrm{k} / 150$ & $0 \%$ & $14 \%$ & $14 \%$ \\
\hline & 4 & $\mathrm{k}$ & 0 & $262 \mathrm{k} / 300$ & $118 \mathrm{k} / 150$ & $-100 \%$ & $-12.7 \%$ & $-21.3 \%$ \\
\hline & 5 & $\mathrm{k}$ & 0 & $137 \mathrm{k} / 300$ & $50 \mathrm{k} / 150$ & $-100 \%$ & $-54.3 \%$ & $-66.7 \%$ \\
\hline
\end{tabular}


TABLE 2 PARAMETERS AND VALUES OF SIMULATION

\begin{tabular}{ll}
\hline \multicolumn{1}{c}{ PARAMETERS } & \multicolumn{1}{c}{ VALUES } \\
\hline Frequency & $0.2 \mathrm{GHz}$ \\
Network scale & $4 \times 4 \times 4$ \\
Topology & 3D Mesh, Cluster Mesh \\
Switching mechanism & wormhole \\
Flow control & Credit-based \\
Routing algorithm & XYZ, TADR, TLAR, DRTL \\
Packet injection model & Exponential distribution \\
Traffic model & Uniform, hotspot, local traffic \\
Virtual channel per port & 2 \\
Buffer length (flit) & 5 \\
Packet length (flit) & 5 \\
Flit length (bit) & 64 \\
Link bandwidth (bit) & 64 \\
\hline \hline
\end{tabular}


TABLE 3 PARAMETERS AND VALUES IN HOTSPOT

\begin{tabular}{ll}
\hline \multicolumn{1}{c}{ PARAMETERS } & \multicolumn{1}{c}{ VALUES } \\
\hline Chip size & $40.48 \mathrm{~cm}^{2}$ \\
Total layer thickness & $0.34 \mathrm{~mm}$ \\
Core power & $27.794 \mathrm{~mW}$ \\
Convection capacitance & $140.4 \mathrm{~J} / \mathrm{K}$ \\
Convection resistance & $0.1 \mathrm{~K} / \mathrm{W}$ \\
Heat sink side & $60 \mathrm{~mm}$ \\
Heat sink thickness & $6.9 \mathrm{~mm}$ \\
Specific heat capacity in layer0 & $1.75 \mathrm{E}+06 \mathrm{~J} /\left(\mathrm{m}^{3} \mathrm{~K}\right)$ \\
Specific heat capacity in layer1 & $4.00 \mathrm{E}+06 \mathrm{~J} /\left(\mathrm{m}^{3} \mathrm{~K}\right)$ \\
Specific heat capacity in layer2 & $1.75 \mathrm{E}+06 \mathrm{~J} /\left(\mathrm{m}^{3} \mathrm{~K}\right)$ \\
Specific heat capacity in layer3 & $4.00 \mathrm{E}+06 \mathrm{~J} /\left(\mathrm{m}^{3} \mathrm{~K}\right)$ \\
\hline \hline
\end{tabular}

